\title{
Smart Home Energy Management - the Future of Energy Conservation: A Review
}

\author{
Yadu Krishnan \\ Dept. of Mechanical Engineering \\ Sir M. Visvesvaraya Institute of Technology, VTU \\ Bengaluru, India
}

\author{
Anagha S, Apurva Karn, Misha Saara \\ Dept. of Electronics and Communication \\ Sir M. Visvesvaraya Institute of Technology, VTU \\ Bengaluru, India
}

\begin{abstract}
Energy management in households gets increasingly more attention in the attempt to integrate more sustainable energy resources. With the rising cost of energy and the ever-increasing demand, we and our house have to get smart to keep the bills down. Smart grid systems are envisioned to be a part of this effort towards a better utilization of energy production and distribution infrastructure. The Home Energy Management System (HEMS) can be considered as the foundation in this endeavor. Its main goal is to enable energy management services for efficient monitoring and management of electricity generation, power conservation, as well as energy storage methods designed within the smart home. With the role of being both, an essential link in transmission infrastructure for balancing the electric grid and a surveillance unit in private homes, the technology used becomes essential to address. This review gives an insight on how the smart grid combines artificial intelligence, computing, communication and Internet technologies to create a smarter power system. It enables in producing better power quality and lower generation cost in comparison to the conventional power grid transmission system. The study will discuss about the development of HEMS associated with smart grid technology distinguishing its features from the traditional methods and hence suggesting a framework for improving the power losses and the voltage profile.
\end{abstract}

Keywords- Energy, management, technology, smart grid, transmission, conservation, HEMS

\section{INTRODUCTION}

The asymptotic rise in technology has revolutionized the lifestyle of humanity and changed our world into a digital era. With the increased usage of electronic gadgets in our day to day life, the demand for sustainable power supply/electrical energy is always at its peak. The constant growth in the need for power has raised the cost of electricity per unit consumption. India is truculent to meet the electric power demands of a fast expanding economy. Reconstructing of the power industry has only increased several challenges. The growth in demand for energy is increasing which may anytime exceed the implementation of conventional energy generation systems significantly. This scientific assumption has raised the alarm among the energy sectors in the world which motivated the researchers to propose alternatives like mystification of energy sources such as coal, natural gas oils, fossil fuels, etc., but their use has resulted in negative impact over the environment, more expensive and high risk. India suffers from serious power shortage which is likely to worsen over the next few decades. It has a power sector characterized by deficient generation and high distribution losses. This resulted in a shift of focus area of the scientists towards evolving strategies that aimed at saving and developing an alternative source of power generation. The current era of launching new electrical and electronic devices has an impact on the quality of lifestyle led by people and has significantly increased the demand towards maintaining a sustainable usage of electrical energy for household consumption. Intelligent systems driven by microprocessors and computers need to be employed for online monitoring and control of modern large-scale power systems, to overcome the complexities and drawbacks of the conventional instrumentation schemes. These intelligent systems form the basis of the smart grid by itself does not completely solve the problem of the existing demand-supply mismatch. Therefore, there is a need for Home Energy Management System (HEMS). The core functionality of a versatile HEMS involves the use of electricity within the home. It gives the user the ability to 'see' what devices are doing and to remotely 'reach in' and turn them on \& off or otherwise modify their operation. Generally, the conservation of domestic energy is done in a sporadic and non-autonomous manner. The automation of energy consumption yields optimization benefit in a significant manner. The HEMS implemented at home uses devices such as electrical sensors, relays, data network, and flexible computing platform, that guarantees an efficient management process. The focus of HEM is towards prioritizing the load consumption concerning the availability of both cost and energy.

\section{ELECTRICAL GRID}

Electrical grid or power grid is an intricate, interconnected network designed to deliver electricity from the producers to the consumers, who then use it for their daily needs. These have grown from small local designs, to stretching thousands of kilometers and connecting millions of homes and businesses today. The grid system consists of countless complex interconnections, however there are three main sections.

1.Electricity generation

2.Transmission

3.Distribution

\section{A. Conventional Grid System}

The traditional electric power grid connected large central generating stations through a high voltage (HV) transmission system to a distribution system that directly fed customer demand. Generating stations consisted primarily of steam stations that used fossil fuels and hydro turbines that turned high inertia turbines to produce electricity. The transmission system grew from local and regional grids into a large 
interconnected network that was managed by coordinated operating and planning procedures.

The drawbacks of this system such as its aging infrastructure and its inability to accommodate small scale renewable energy sources has led to some technical advances to meet the supply-demand equilibrium. However, the technological advances continually updated to the bulk power grid such as using High Voltage Direct Current (HVDC), Flexible Alternating Current Transmission Systems (FACTS) and distributed generation systems have proved to be futile attempts in modernizing the grid system.

The basic grid setup is as shown below. In reality there are many more of each system connected to the grid, however, for a conceptual grasp this image should demonstrate how interconnected even a simple grid may be.

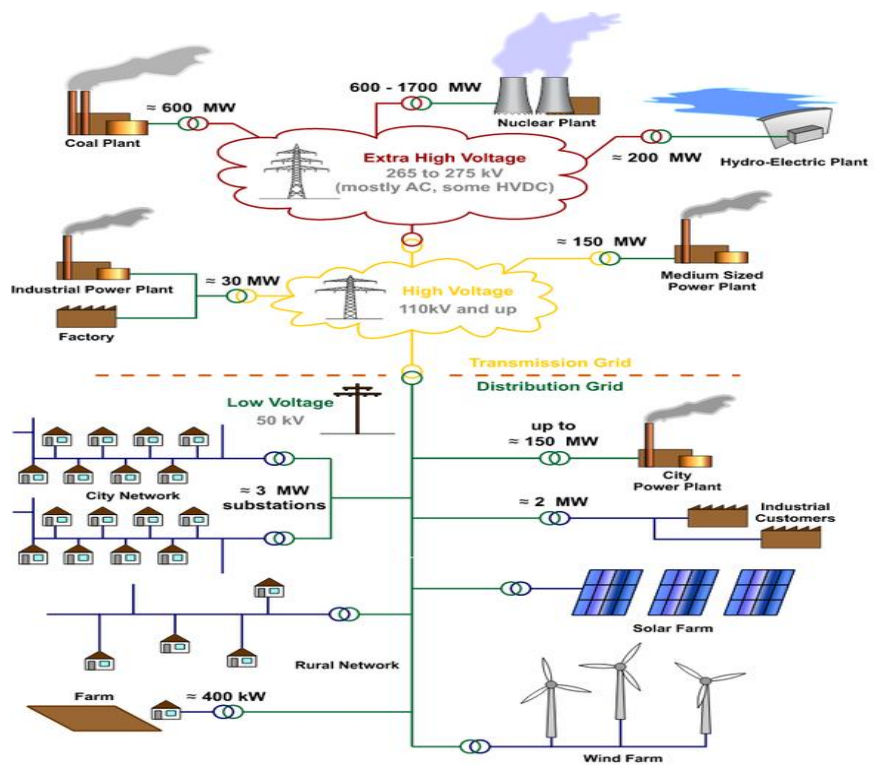

Fig 1: Basic Layout of an Electrical Grid

\section{B. Smart Grid System}

The drawbacks of the traditional grid system and its advances over recent years has led to the advent of the concept of the smart grid. The smart grid system is a potential solution to these issues. A smart grid is an enhancement of the 20th century electrical grid, using two-way communications and a variety of operation and energy measures including smart meters, smart appliances, renewable energy resources and energy efficient resources. Two-way flows of electricity and information could improve the delivery network.

A smart grid would allow the power industry to observe and control parts of the system at higher resolution in time and space. One of the purposes of the smart grid is real time information exchange to make operation as efficient as possible. It would allow management of the grid on all time scales from high-frequency switching devices on a microsecond scale, to wind and solar output variations on a minute scale, to the future effects of the carbon emissions generated by power production on a decade scale.

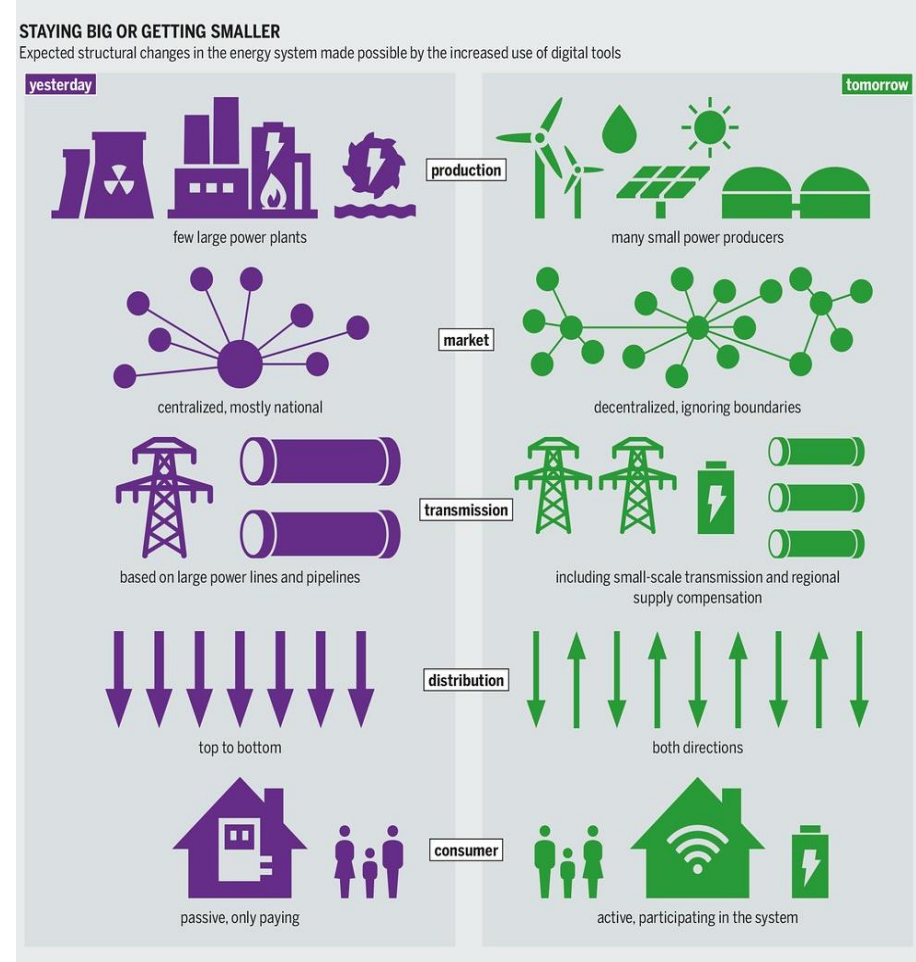

Fig 2: Characteristics of a Traditional System vs Smart Grid System

\section{HOME ENERGY MANAGEMENT SYSTEM}

\section{Concept of HEMS}

A Home Energy Management System is a technology platform comprised of both hardware and software that allows the user to monitor energy usage and production and to manually control and/or automate the use of energy within a household. Beneath the umbrella of grid architecture, Advanced Metering Infrastructure (AMI) devices have activated a dependable communication mode connecting both power utilities and residential consumers. This communication channel paved the way for an opportunity to include the concepts of economic incentives conceptualized for a smart home for managing the demand-side resource by switching to and from their energy consumption during peakload hours of the day as a method to shed load for reduced electricity bills. The key element that allows all of the emerging Smart Grid technologies to function together is the interactive relationship between the grid operators, utilities, and users.

The main objective of the use of HEMS is to enable the consumer to monitor and control the amount of energy consumed or to consume it in a more efficient way. For this, the consumer should know how the energy is being used in his home which can only be computed when energy throughout the home is monitored. The basic elements of a smart home are as shown in Fig 3. [1] 


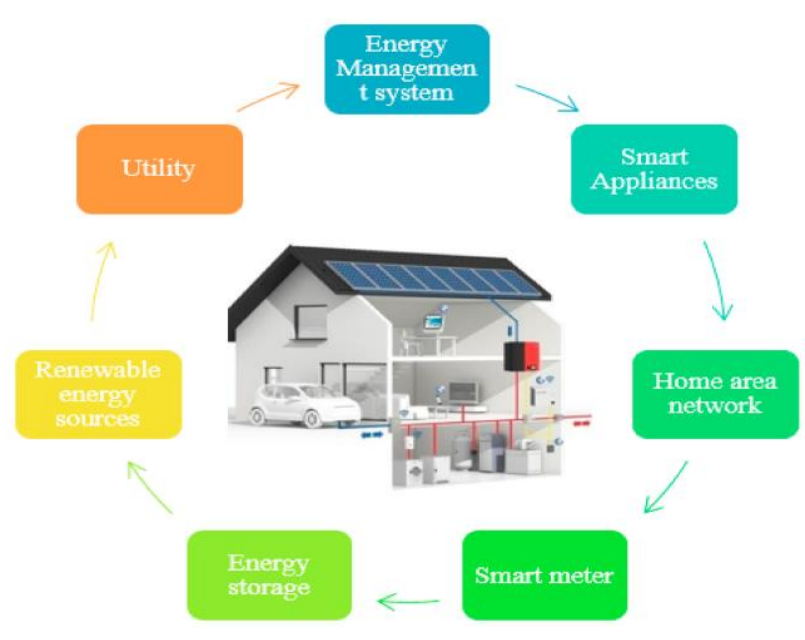

Fig 3: Elements of a Smart Home

\section{Functionalities of HEMS}

The main goal of HEMS is to improve energy efficiency in homes and buildings. Additional goals may include electric utility benefits, such as controlling energy usage to reduce peak demand and support load shifting. To achieve these goals, the HEM needs to have certain functionalities and features explained below and shown in Fig 4.

- Monitoring: HEMS need to be able to monitor and control different devices and appliances in a home. the process of monitoring makes real-time information regarding energy usage pattern accessible. Device information can be available to the user via either a web interface or phone/tablet application.

- Logging: Logging is the process of recording data information pertaining to the unit of electricity consumed by each appliance. This functionality includes analysing demand response (DR) for realtime prices. For better DR support, information for multiple homes in a community needs to be available, and the system needs to be able to use an optimization method to intelligently respond to DR signals and allocate resources to the homes efficiently.

- Control: In its simplest form, device control should be available to the user manually. If the management system supports smart scheduling, control can be automatic. Moreover, control of devices can be remote or local.

- Management: In the smart grid era, information regarding energy usage can be provided at different granularities from a wide variety of devices. HEMS should be capable of handling very large amounts of data efficiently.

- Alarm: Here alarms are generated as well as passed on to the smart HEMS centre which contains information regarding fault locations, types, etc. [1][2]

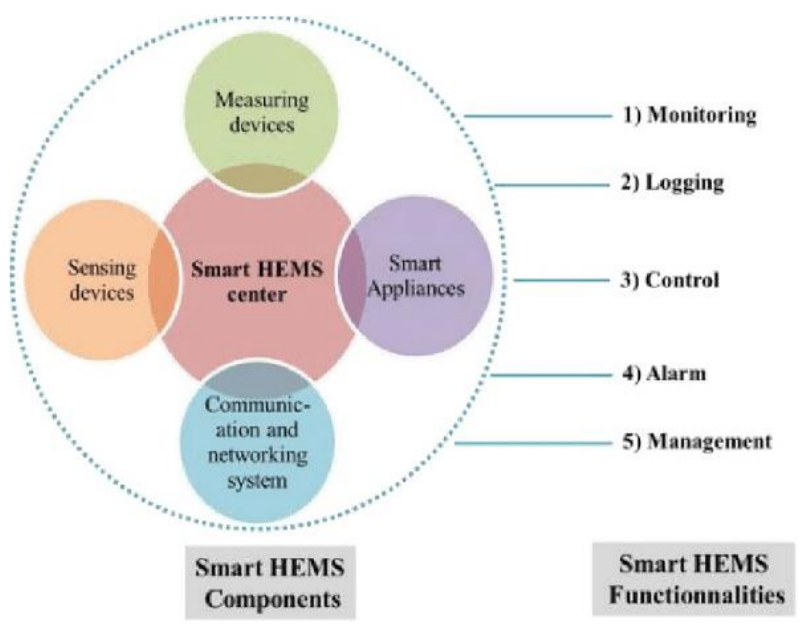

Fig 4: Functionalities of HEMS

\section{Architecture of HEMS}

A home energy management system's hardware usually consists of a 'hub' device which relays communications between the on goings inside the house, the user and in some cases, the local utility or electricity retailer. This hub is usually installed on the home's electrical board, but may also be installed 'virtually' in cases where the HEMS operates purely on a wireless network. Other less essential components may include 'smart plugs ', light \& temperature sensors, and smart devices within the home. The software used in a HEMS is what moderates the incoming and outgoing data and communications. From a user's perspective, the software is the interface that allows access to monitoring data and control functions of the system. The interface usually takes the form of an app or web portal. In many cities across the nation, new equipment, appliances, and software are available that use emerging Smart Grid technologies to save energy, seek out the lowest rates, and contribute to the smooth and efficient functioning of our electric grid. The architectures, proposed by researchers in different articles may be categorized under various heads, considering criteria related to the structure of monitoring, management, and distribution and communication capabilities. The overall architecture is as shown in Fig 5.[1][2]

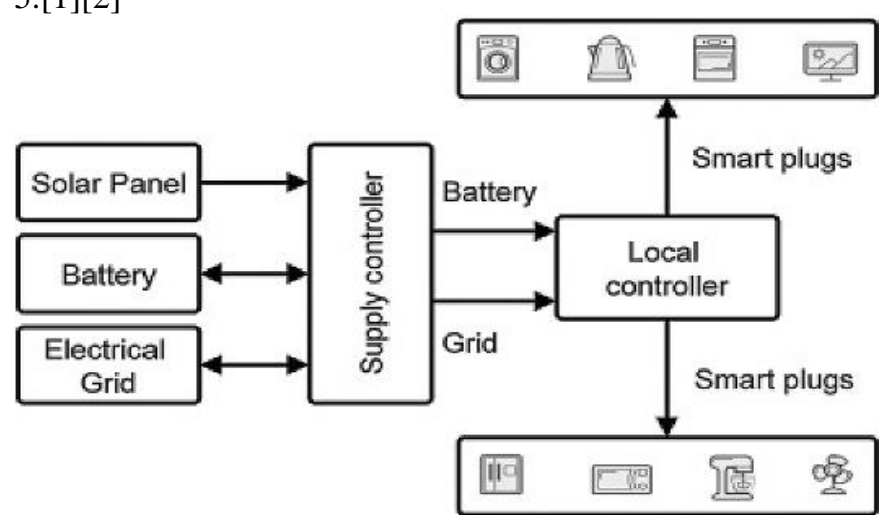

Fig 5: Overall architecture of a representative HEM 
HEMS architecture typically consists of components as mentioned below-

- Monitoring and control devices: These devices carry out the monitoring and controlling of energy utilization for various appliances

- Processor: Processor is used for concentrating, storage and management of data and information. Here, the server and the database are located in the central module.

- Gateway: Gateway allows the network between HEMS and the outside world, to facilitate remote access via Internet.

\section{Proposed HEMS}

\section{A. Smart Home Energy Management System}

The proposed Smart Home Energy Management System consists of Home Smart Gateway (HSG), Sensing Units (SU), and End Appliance Unit (EAU). Fig 6 shows the essential architecture of the proposed SHEMS. It represents the gateway of the whole system to the external world. The home gateway (H-Gateway) is a single chip embedded system integrated with GSM modem and it is installed at the consumer premises. The utility server (U-Server) is a highend $\mathrm{PC}$ and it is installed at the utility headquarter. An adaptive algorithm was designed to be running continuously to elicit scheduled operation for the appliances according to the activity behaviour of the home residents. The HSG is responsible for monitoring all the Sensing Units (SUs) in the home and controlling the End Appliance Units (EAUs). Therefore, the HSG is designed to monitor and control all sensors and appliances through the local Wi-Fi network by using Wi-Fi module. In addition, GSM modem is employed to provide a link between the residents when they are outside the home for monitoring and control purposes. The GSM modem and the Wi-Fi module have been interfaced to Microcontroller Unit (MCU) via UART. The microcontroller used in the proposed system is PIC18F4620 from Microchip Inc. For the Wi-Fi network connection, the ESP8266 module is interfaced to the MCU in HSG and to the other units, EAUs and SUs. On the other hand, SIM900 GSM modem is used to secure the external connection of HSG to the GSM network. Here, the resident could control and monitor any appliance remotely using SMS. Furthermore, HSG is programmed to have the ability of controlling the operation of lighting for each room separately depending on its motion. [3]

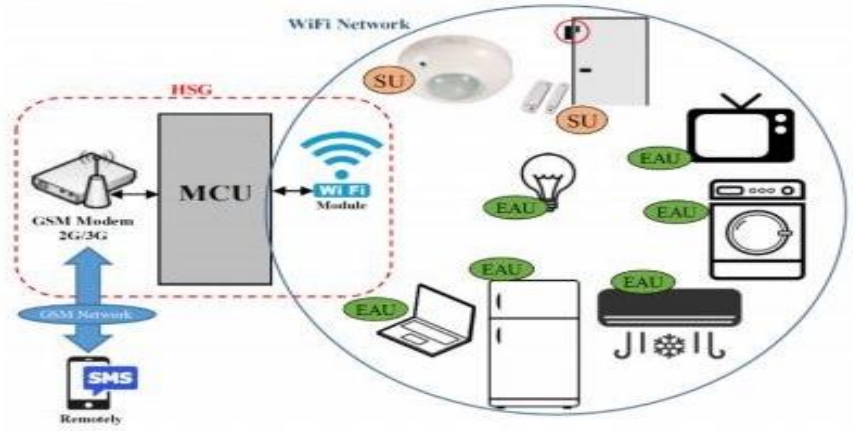

Fig 6: Proposed SHEMS Architecture

\section{B. IoT Based HEMS}

Another system as shown in Fig 7 is proposed which uses IoT to overcome the disadvantages of the conventional HEMS. The user-centric service domain is defined as the service domain where the user-centric services are provided autonomously, based on the contextual information related to users and environments. When the user with a NA enters the service domain, the networked devices (e.g., networked appliances, networked lighting systems, smart meters, and networked PV systems) in HANs (Home Area Networks) exchange data via the NA, by constructing peer-to-peer (P2P) connections. The important feature of the proposed HEM is that the HAN can be dynamically configured by using the user's NA, without any fixed GWs (Gateways). In other words, instead of the conventional GWs in the HAN, the NA is in charge of the role of interconnection between a CMS and an end device. Another advantage of this system is not only reducing the cost of system construction and management, but also providing user-centric services to users through the NA. In the proposed system, the mobile device is used as a service provider, as well as the network resources. The Bluetooth technology for communications is used because recently most mobile devices have adopted Bluetooth as a main Wireless Personal Area Network (WPAN). In this way, it is possible to construct HANs without installing additional GWs.[4]

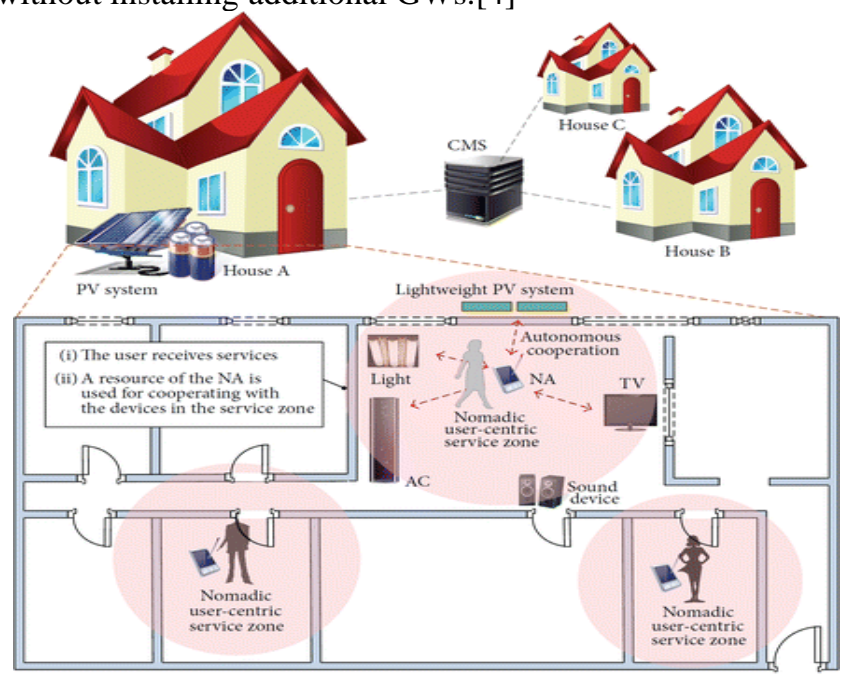

AC: air condittoner

Fig 7: IoT Based Proposed HEMS

\section{DRAWBACKS OF HEMS}

The future seems only a few steps away with HEMS being able to monitor our energy usage and help us save bills. But at the same time, the proposed system has its own set of disadvantages and they are as follows-

- Capital Outlay: The main disadvantage is the high cost of setting up a fully functional smart home.

- Integration: Designing an integrated platform that will make the appliances working under different standards interoperable is an open research issue. Also, the simplicity and intuitiveness of this user 
interface will be of paramount importance for the success of HEMS.

- Technology Learning Curve: As much as we claim to be in the digital age, it is undeniable that many people are still relatively unfamiliar with technology. The population of such individuals increases as we move up the age pyramid. And this becomes more of a problem since one of the major arguments of the proponents of smart homes is the convenience it offers to the elderly.

- Security and Privacy: We are aware that any system connected to the Internet and completely run by a computer is prone to being hacked. In other words, cyber-attacks. Privacy also remains another recurrent disadvantage. Since the system is connected to the Internet, anyone could hack into the video feed (if a camera is setup in the house).

- Signal speed: The speed of the communication signals should be higher. This has no negative effects on the performances of other devices in the system. But providing the system with such properties will need to advance the technology with high cost.

\section{CONCLUSION}

This study first presents a comprehensive overview on how crucial energy management is, for the benefit of the greater good. It gives an insight on how the traditional grid system, despite the prevailing drawbacks of its advancements can only lead to multiple challenges as the demand grows further into the future. With the advent of smart grid and its advancements, greater awareness can be brought to the masses to switch to a more efficient, clean and green system. This is made possible through the use of Home Energy Management Systems which provide different functionalities in order to monitor, control and manage energy usage. Different kinds of HEMSs are proposed out of which the IOT based Home Energy Management System proves to be the better choice. In spite of the drawbacks that can be corrigible, smart home management systems appear to be a tangible solution to the energy crisis we face today.

\section{FUTURE SCOPE}

Smart grids are not only aligned perfectly with the needs and demands of our time, they are also predicted to have significant long-lasting effects. For instance, the technology will overhaul aging equipment and bring things up to speed. This will help to reduce the likelihood of blackouts, burnouts and power surges. This technology will also reduce both, the cost of energy consumption, production and the need of the grid storage. With its full implementation, smart grids will make renewable power feasible and equip the grid to meet increasing energy demands. It provides the user real-time control over their electricity bills. It can help reduce greenhouse gas emissions by up to 211 million metric tons and is much more reliable than a traditional grid. Research will focus primarily on machine learning, plug and play technology, self-healing and total automation of the grid. These leverage developments in sensing, communication and computational technologies to improve the reliability and efficiency of the system. In addition, newer technologies and future-proof solutions such as artificial intelligence to handle huge blocks of user data), internet of things, virtual/augmented reality, and automation are becoming integral parts. This contributes towards the 'Digital India' scheme initiated by the Government of India to transform into smart cities. Thus, every node in the power network of the future will be awake, responsive, adaptive, price-smart, eco-sensitive, real-time, flexible, humming and interconnected with everything else.

\section{REFERENCES}

[1] Bandana Mahapatra, Anand Nayyar, "Home Energy Management System (HEMS): Concept, Architecture, Infrastructure, Challenges and Energy Management Schemes", Energy Systems, 2019.

[2] Helia Zandi, Teja Kuruganti, Edward A Vineyard, David Fugate, "Home Energy Management Systems: An Overview", 9 International Conference on Energy Efficiency in Domestic Appliances and Lighting, 2018

[3] Bilal Mubdir, Asaad Al-Hindawi, Noor Hadi, "Design of Smart Home Energy Management System for Saving Energy", European Scientific Journal, Vol 12, No. 33, November 2016

[4] Jongbae Kim, Jinsung Byun, Daebeom Jeong, Myeong-in Choi, Byeongkwan Kang, Sehyun Park, "An IoT-Based Home Energy Management System Over Dynamic Home Area Networks", International Journal of Distributed Sensor Networks, 2015.

[5] Mohammadreza Daneshvar, Mahmoud Pesaran, Behnam Mohammadi-ivatloo, "Transactive Energy in Future Smart Homes", The Energy Internet, 2019

[6] Yonghong Ma, Baixuan $\mathrm{Li}$, "Hybridized Intelligent Home Renewable Energy Management System for Smart Grids", Sustainability, 2020.

[7] Hartono BS, Sri Paryanto Murshid, Sapto Prajogo, "Review: Home Energy Management System in a Smart Grid Sceme to Improve Reliability of Power Systems", $2^{\text {nd }}$ International Tropical Renewable Energy Conference, 2017.

[8] Soren Aagard Mikkelsen, Rune Hylsberg Jacobsen, "Securing the Home Energy Management Platform”, 2015. 